\title{
A review of chronic lung disease in neonates at Charlotte Maxeke Johannesburg Academic Hospital from 1 January 2013 to 31 December 2014
}

\author{
A V Mphaphuli, MB BCh, DCH; D E Ballot, MB BCh, FCPaed, PhD \\ Department of Paediatrics and Child Health, University of the Witwatersrand and Charlotte Maxeke Johannesburg Academic Hospital, \\ Johannesburg, South Africa
}

Corresponding author: A V Mphaphuli (mphaphulia@gmail.com)

\begin{abstract}
Background. Chronic lung disease (CLD) remains a significant morbidity in preterm babies despite advances in neonatal care. The use of postnatal corticosteroids (PNCSs) to treat CLD remains controversial.

Objectives. To describe the clinical characteristics of babies with CLD at Charlotte Maxeke Johannesburg Academic Hospital (CMJAH) and to explore the use of PNCSs for the prevention and treatment of CLD.

Methods. This was a 2-year retrospective review of neonates admitted to CMJAH. Neonates who were in hospital for $\geq 28$ days were included. Comparisons were made between neonates with evolving CLD and those with no CLD.

Results. A total of 485 neonates were analysed: 237 had evolving CLD and 245 did not have CLD. Overall incidence of evolving CLD was $5 \%$. More neonates with CLD than those without CLD needed resuscitation at birth $(48.5 \%$ v. $39.8 \% ; p=0.02)$ and had low 5 -minute Apgar scores (17.2\% v. 10.6\%; $p=0.001)$. Neonates with CLD had increased prevalence of patent ductus arteriosus $(30.4 \%$ v. $7.7 \% ; p=0.001)$ and late-onset sepsis $(56.5 \%$ v. $23.6 \% ; p=0.001)$. The mortality rate was also higher in CLD babies $(10.2$ v. $2.4 \% ; p=0.001)$. Necrotising enterocolitis (NEC) $(29.2 \%$ v. $8 \%$; $p=0.005)$ and sepsis $(83.3 \%$ v. $53.8 \% ; p=0.008)$ were associated with increased mortality. The use of PNCSs was associated with less NEC (3.5\% v. $17.2 \%$; $p=0.001)$ and improved survival $(95.6 \%$ v. $81.7 \% ; p=0.001)$.

Conclusions. CLD remains a common morbidity in neonates despite advances in neonatal care. The use of PNCSs was shown to have short-term benefits. To get the most out of PNCS use for CLD, further studies need to be conducted to determine the safest type of steroid, safe doses and the duration of treatment.
\end{abstract}

S Afr J Child Health 2016;10(3):161-165. DOI:10.7196/SAJCH.2016.v10i3.1060

The clinical definition of neonatal chronic lung disease (CLD), also known as bronchopulmonary dysplasia (BPD), has evolved over time. It was first defined by Northway et al.$^{[1]}$ in 1967 as persistent respiratory signs and symptoms along with the need for supplemental oxygen and an abnormal chest X-ray (CXR) at 28 days of age. The definition of CLD was subsequently modified and defines BPD as oxygen dependence at 36 weeks post-menstrual age (PMA) with or without the use of respiratory support and with or without the characteristic radiographical changes. ${ }^{[2]}$ These definitions do not consider gestational age (GA) and do not indicate the level of oxygen dependence, which can range from needing low-flow oxygen to being ventilator dependent. To address this issue, the National Institute of Health (USA) has developed a consensus severity-based definition. This definition includes all babies born who need more than $21 \%$ supplemental oxygen for at least 28 days. CLD is further classified into mild, moderate and severe, depending on the $\mathrm{FiO}_{2}$ needed and the duration of oxygen therapy for preterm babies. ${ }^{[3]}$ Because of the complexity of the definition, some units just consider the need for oxygen on day 28 of life and often refer to this as evolving CLD. The incidence of CLD as defined by the need for oxygen supplementation at 36 weeks PMA is $\sim 30 \%$ of premature infants with birth weight (BW) $<1000 \mathrm{~g}$; it is uncommon in infants born at $>30$ weeks' gestation or a weight of $>1250 \mathrm{~g}^{[3]}$

CLD has multifactorial aetiology and remains a major cause of morbidity in premature infants. ${ }^{[1]}$ Contributing factors include infection, exposure to high oxygen levels with the formation of toxic oxygen-free radicals and ventilator-induced lung injury that results in arrested lung development and impaired lung function $\cdot^{[4]}$ Several maternal risk factors, including increasing age, hypertension, lack of antenatal steroid use and chorioamnionitis have been associated with BPD. ${ }^{[4]}$
The management of CLD includes several preventive and therapeutic strategies that target several pathways and processes involved in the pathogenesis of CLD. Some provide antioxidant protection; others minimise specific aspects of inflammation, reduce elastolytic and proteolytic injury, or regulate growth. In addition, supportive pharmacological treatments that target the development of pulmonary oedema, bronchoconstriction and impaired gas exchange are used. The success of these interventions has been variable. ${ }^{[5]}$

Postnatal corticosteroids (PNCSs) have been extensively studied and have been found to be effective in weaning infants off mechanical ventilation. ${ }^{[6-8]}$ This effect has been proven for dexamethasone, which is the most widely studied PNCS in randomised controlled trials (RCTs). Despite the short-term benefits, dexamethasone has not been shown to reduce the total days of hospitalisation, duration of supplemental oxygen therapy, or incidence of CLD. ${ }^{[6-8]}$ In the era before PNCS treatment, the long-term neurodevelopmental outcome for survivors with CLD was worse than that in similar infants without CLD. ${ }^{[9]}$ However, adverse effects of PNCSs, including hyperglycaemia, gastrointestinal tract (GIT) perforation, hypertension, infection, steroid-induced cardiomyopathy, long-term neurodevelopmental effects and growth retardation complicate the use of PNCSs. The most worrisome long-term effect is increased risk for poor neurological outcome, including cerebral palsy (CP). Corticosteroids can have direct toxic effects on the developing brain, including neuronal necrosis, interference with healing and inhibition of brain growth. ${ }^{[9,10]}$ A systematic review showed a significantly higher rate of $\mathrm{CP}$ after corticosteroid treatment and a non-significant reduction in mortality. ${ }^{[11]} \mathrm{A}$ multicentre, double-blinded RCT testing early postnatal dexamethasone therapy for prevention of CLD had to be stopped before completion because of concern about 
serious side-effects such as GIT perforation and periventricular leukomalacia (PVL) ${ }^{[12]}$ The American Academy of Pediatrics (AAP) also recommended that alternative corticosteroids undergo studies and that all infants enrolled in RCTs for PNCSs receive long-term neurodevelopmental follow-up. ${ }^{[13]}$

Owing to concern about the safety of PNCSs, in 2002 the AAP released a policy statement regarding the use of PNCSs for prevention or treatment of CLD, stating that the routine use of dexamethasone could not be recommended. ${ }^{[13]}$ The AAP recommended that dexamethasone use be limited to RCTs with long-term follow-up. Since the publication of the AAP statement, postnatal use of dexamethasone for CLD has reduced; however, the incidence of CLD has not diminished. Some reports have suggested that the incidence and severity of CLD may have actually increased. ${ }^{[14]}$

The data available for PNCS use in CLD are inconclusive and conflicting. As a result, clinicians are advised to use their own clinical judgement to balance potential adverse effects of CLD with the potential adverse effects of PNCS for each individual patient.

The incidence of CLD in very-low-birth-weight (VLBW) babies at Charlotte Maxeke Johannesburg Academic Hospital (CMJAH) is lower than that reported in the Vermont Oxford Network (VON) ${ }^{[15]}$ At $\mathrm{CMJAH}$, babies who are on supplemental $\mathrm{O}_{2}$ for $>28$ days are given oral prednisolone (OP) for prevention/treatment of CLD. Babies who remain ventilator dependent are given dexamethasone.

Alternative PNCSs include hydrocortisone, nebulised dexamethasone and $\mathrm{OP}^{[5]}$ One study looking at the effect of a short course of OP in infants with $\mathrm{O}_{2}$-dependent BPD provided evidence that OP is effective in select patients with BPD ${ }^{[16]}$ Characteristics of babies with CLD and the use of PNCSs have not been reviewed at CMJAH. This study aimed to describe babies with evolving CLD and to explore the use of PNCSs for evolving CLD at CMJAH. The objectives were to determine the incidence of CLD at CMJAH, to describe clinical and demographic characteristics and survival to discharge in babies with CLD, and to compare these with those of babies without CLD.

\section{Methods}

This study was an institution-based retrospective audit conducted in the neonatal unit at CMJAH in Parktown, Gauteng Province, South Africa. Evolving CLD was defined as oxygen use at 28 days of life; the need for supplemental oxygen at 36 weeks PMA was considered as definite CLD (VLBW babies only). Following approval by the Committee for Research in Human Subjects at the University of the Witwatersrand (Medical), a 2-year (1 January 2013 - 30 December 2014) retrospective review of neonatal medical records was performed. Data from the existing CMJAH neonatal database (Research Electronic Data Capture hosted by the University of the Witwatersrand) were used for analysis. ${ }^{[17]}$ The data were collected from clinician-completed hospital records, prospectively on an ongoing basis, for the purpose of clinical audit. All babies admitted to the CMJAH neonatal unit within 72 hours of life (inborn and outborn), with BWs of $\geq 500 \mathrm{~g}$ and who were in hospital for $\geq 28$ days were included. This group was divided into babies with evolving CLD and those without. The neonatal unit at CMJAH has 88 beds, 35 of which are high care, 14 in the paediatric/neonatal intensive care unit, 20 low care and 15 kangaroo mother care. Respiratory support includes early rescue surfactant (SVT), supplemental oxygen via low-flow nasal cannulae $\left(\mathrm{NPO}_{2}\right)$, nasal continuous positive airway pressure ventilation (NCPAP), intermittent positive-pressure ventilation (IPPV) and high-frequency oscillatory ventilation. Owing to limited resources, ventilatory support in the form of NCPAP was only offered to babies with $\mathrm{BW} \geq 750 \mathrm{~g}$ who showed signs of respiratory failure. Babies with $B W \geq 900 \mathrm{~g}$ who showed signs of respiratory failure on NCPAP or who became apnoeic were offered IPPV. Respiratory failure was defined as $\mathrm{O}_{2}$ saturation $<88 \%$ on $60 \%$ supplemental $\mathrm{O}_{2}$, respiratory acidosis ( $\mathrm{pH}<7.25$ with $\mathrm{PaCO}_{2}>60 \mathrm{mmHg}$ ) or markedly increased work breathing.

\section{Definitions}

Maternal hypertension included both chronic and pregnancyinduced hypertension. Chorioamnionitis was defined as premature and/or prolonged rupture of membranes, fever and foul-smelling liquor in mothers. Resuscitation at birth was defined as the need for bag mask ventilation, chest compressions, or intubation and ventilation. The Ballard score was used to estimate gestational age (GA). Fenton growth charts (2013) were used to assess weight for gestational age ${ }^{[18]}$ The whole group of neonates was described and then stratified by BW category, namely:

- $\geq 500$ - 999 g, extremely low birth weight (ELBW)

- $\geq 1000$ - 1499 g, very low birth weight (VLBW)

- $\geq 1500$ - $2499 \mathrm{~g}$, low birth weight (LBW)

- $\geq 2500$ g, normal birth weight (NBW).

Babies were considered to be small for GA if the BW was <10th percentile. ${ }^{[18]}$ The 5-minute Apgar scores were categorised into two groups, namely Apgar score $\leq 5$ and $>5$. Babies were divided according to GA into two groups, namely $<32$ weeks and $\geq 32$ weeks. PNCSs were defined as steroids given in an attempt to facilitate weaning of patients off prolonged ventilation or supplemental oxygen. Dexamethasone was given to patients failing to wean off mechanical ventilation. NEC was considered as modified Bell's stage $\geq 2 .{ }^{[19]}$ Sepsis was classified as culture-proven bacterial or fungal blood stream sepsis only.

\section{Statistical analysis}

The data were assessed for missing information and erroneous or suspicious entries. These entries were verified as far as possible with the original patient records. Information not available from the database was obtained from hospital files drawn from the hospital record archives. The database was then exported to SPSS Statistics version 23.0 (IBM Corp., USA) for analysis.

\section{Babies with evolving CLD}

Babies in different weight categories were compared in terms of therapeutic intervention and outcome. Babies who received PNCSs were compared with those who did not, and babies who survived to discharge were compared with those who died. Finally, the CLD group was then compared with the no-CLD group. The data were normally distributed, so continuous variables were described using means and standard deviations (SDs), while frequencies (percentages) were reported for categorical variables. For comparison, $\chi^{2}$ tests were used for categorical variables and independent $t$-tests for continuous variables. All analyses considered a value of $p<0.05$ as significant.

\section{Results}

There were 485 babies hospitalised for more than 28 days; records were not retrievable for 3 patients. Therefore, 482 patients were included, 237 with evolving CLD and 245 without CLD.

The overall incidence of evolving CLD was $5.1 \%$. The incidence in the VLBW babies was 206/1 $302(15.8 \%)$ and $31 / 3268(0.94 \%)$ in the $>1500 \mathrm{~g}$ babies $(p<0.0001)$. The incidence of definite CLD was 98/1 $302(7.5 \%)$ in the VLBW babies.

Demographic and birth characteristics are shown in Table 1. There was no difference in the mean (SD) BW between babies with and without CLD (1 017 (101) g v. 1041 (104) g, p=0.57). Similarly, gestational age was not different between the two groups (CLD 28.2 (1.9) weeks v. no CLD 28.3 (1.9) weeks, $p=0.9$ ). There were, however, more babies who were SGA in the no-CLD group than in those with CLD (26.5\% v. $12.9 \%$, $p=0.03)$. The percentage of males with CLD was greater (50.2\%) than in the no-CLD group (40.8\%). There was no significant difference in maternal obstetric and labour-room characteristics between CLD and 
no-CLD babies (Table 1). There were more babies who had 5-minute Apgar scores $\leq 5$ and who needed resuscitation at birth in the CLD group.

Therapeutic interventions, morbidity and outcome are shown in Table 2. Neonates in the CLD group were more likely to be ventilated and to receive surfactant than those without CLD. The duration of ventilation was significantly longer in babies with CLD compared with those without CLD (15.7 (16.0) days v. 6.1 (2.8) days, $p=0.001$ ).
Table 1. Demographic, obstetric and labour room characteristics

\begin{tabular}{lllll}
\hline & CLD, $\boldsymbol{n}$ (\%) & No CLD, $\boldsymbol{n}(\%)$ & $\chi^{2}$ & $p$-value \\
\hline GA for BW, SGA & $30 / 232(12.9)$ & $65 / 243(26.5)$ & 5.911 & 0.030 \\
Gender, male & $118 / 235(50.2)$ & $100 / 245(40.8)$ & 5.911 & 0.016 \\
Maternal characteristics & & & & \\
$\quad$ Black & $221 / 234(94.4)$ & $237 / 244(97.1)$ & 0.038 & 0.845 \\
Antenatal steroids & $93 / 185(50.2)$ & $116 / 219(52.9)$ & 0.658 & 0.429 \\
Maternal hypertension & $51 / 206(24.7)$ & $64 / 220(29.0)$ & 1.014 & 0.328 \\
Chorioamnionitis & $7 / 188(3.7)$ & $9 / 223(4.0)$ & 0.150 & 0.801 \\
Mode of delivery, CS & $133 / 205(64.8)$ & $160 / 241(66.3)$ & 0.017 & 0.896 \\
Apgar score at 5 minutes, $\leq 5$ & $37 / 214(17.2)$ & $26 / 230(10.6)$ & 85.448 & 0.001 \\
Resuscitation at birth & $100 / 197(48.5)$ & $92 / 231(39.8)$ & 5.139 & 0.025 \\
CS = caesarean section. & & & &
\end{tabular}

Table 2. Therapeutic intervention, morbidity and outcome characteristics

\begin{tabular}{lllll}
\hline & CLD, $\boldsymbol{n}$ (\%) & No CLD, $\boldsymbol{n}$ (\%) & $\chi^{2}$ & $\boldsymbol{p}$-value \\
\hline Therapeutic intervention & & & & \\
NCPAP & & & & \\
Ventilation & $120 / 205(58.5)$ & $149 / 237(62.8)$ & 0.09 & 0.696 \\
$\quad$ Postnatal corticosteroids & $114 / 193(59.0)$ & $13 / 230(5.6)$ & 129.07 & 0.001 \\
$\quad$ Surfactant at anytime & $201 / 232(86.6)$ & $174 / 243(71.6)$ & 16.138 & 0.001 \\
Morbidity & & & & \\
$\quad$ Necrotising enterocolitis & $23 / 235(9.4)$ & $14 / 244(5.7)$ & 3.323 & 0.090 \\
Late-onset therapy & $133 / 206(56.5)$ & $58 / 245(23.6)$ & 54.934 & 0.001 \\
PDA & $69 / 220(30.4)$ & $19 / 244(7.7)$ & 41.508 & 0.001 \\
Periventricular leukomalacia & $3 / 187(1.5)$ & $1 / 131(0.5)$ & & \\
Outcome & & & & \\
$\quad$ Mortality & $24 / 236(10.2)$ & $6 / 245(2.4)$ & 12.252 & 0.001 \\
$\quad$ Need for home oxygen & $5 / 219(2.2)$ & $0(0.0)$ & & \\
${ }^{*}$ NCPAP = nasal continuous positive airway pressure ventilation. & & &
\end{tabular}

Similarly, CLD had an increased duration of NCPAP (5.4 (8.7) days v. 2.9 (6.1) days, $p=0.001)$ and hospitalisation (56.0 (20.7) days v. 42.2 (15.1) days, $p=0.001$ ) compared with babies without CLD. However, very few $(2.3 \%)$ of these neonates with CLD needed home oxygen therapy. Mortality was significantly higher in babies with CLD ( $10.2 \%$ v. $2.4 \%, p=0.001)$ and those neonates with CLD were more likely to have a patent ductus arteriosus (PDA) (30.4\% v. $7.7 \%$, $p=0.001)$ and late-onset sepsis (LOS) $(56.5 \%$ v. $23.6 \%, p=0.001$ ).

The effect of BW in the CLD babies is shown in Table 3. In-hospital mortality (overall 10\%) and morbidity in CLD babies was not influenced by BW category. There was, however, a significant difference in the duration of ventilation, with NBW babies staying the longest on the ventilator and VLBW the shortest (31 (21) days v. 12 (12) days). Duration of hospitalisation was longest among the ELBW infants (Table 3).

The use of PNCSs v. no PNCSs in babies with evolving CLD is shown in Table 4. The overall use of PNCSs was high (55\%). PNCSs were associated with less NEC and a lower mortality; however, the duration of ventilation tended to be longer in babies who received PNCSs.

The effect of morbidity and ventilatory support in babies with evolving CLD is shown in Table 5. The incidence of LOS and NEC were significantly higher in the patients who died. The use of early rescue surfactant (SVT) and NCPAP did not improve survival (Table 5), but IPPV was associated with increased mortality: $70.8 \%$ v. $39.7 \%$ in the non-GLD group.

\section{Discussion}

Our study findings showed that evolving CLD in neonates remains a common morbidity in babies at $\mathrm{CMJAH}$, with an overall incidence of $5.1 \%$. The incidence was much higher in the VLBW babies (15.8\%). Data from the 2013 and 2014 VON show rates of CLD of between 10.8 and $30.7 \%$ among different centres. VON only reports VLBW data; this explains the apparently low incidence of CLD

Table 3. The effect of BW in babies with evolving CLD

\begin{tabular}{|c|c|c|c|c|c|c|c|}
\hline & $\begin{array}{l}\text { ELBW, } n(\%) \\
(n=90)\end{array}$ & $\begin{array}{l}\text { VLBW, } n(\%) \\
(n=116)\end{array}$ & $\begin{array}{l}\mathrm{LBW}, n(\%) \\
(n=21)\end{array}$ & $\begin{array}{l}\text { NBW, } n(\%) \\
(n=10)\end{array}$ & $\begin{array}{l}\text { Total, } n(\%) \\
(N=237)\end{array}$ & $\chi^{2}$ & $p$-value \\
\hline Mortality & $10(11.1)$ & $10(8.6)$ & $2(9.5)$ & $2(20)$ & $24(10.2)$ & 1.450 & 0.690 \\
\hline Need for home $\mathrm{O}_{2}$ & $1 / 90(1.1)$ & $3 / 108(2.6)$ & $0(0.0)$ & $1 / 9(7.1)$ & $5 / 219(2.2)$ & 4.310 & 0.229 \\
\hline Necrotising enterocolitis & $7 / 89(7.8)$ & $11(9.5)$ & $4 / 20(20)$ & $2(20)$ & $24(10.2)$ & 3.797 & 0.284 \\
\hline Patent ductus arteriosus & $30(33.3)$ & $38(32.8)$ & 0 & $1 / 5(7.1)$ & $69 / 220(30.4)$ & 5.147 & 0.161 \\
\hline LOS & $59 / 90(65.4)$ & $57 / 116(49.1)$ & $13 / 20(65)$ & $5 / 10(35.7)$ & $133 / 206(56.5)$ & 6.323 & 0.970 \\
\hline Duration of hospitalisation (days), mean (SD) & $62(17)$ & $53(23)$ & $44(13)$ & $44(16)$ & & & 0.001 \\
\hline Duration of ventilation (days), mean (SD) & $17(19)$ & $12(12)$ & $15(12)$ & $31(21)$ & & & 0.007 \\
\hline
\end{tabular}


Table 4. The effect of PNCS v. no PNCS use in the evolving CLD

\begin{tabular}{|c|c|c|c|c|}
\hline & \multirow{2}{*}{$\begin{array}{l}\text { PNCS }(n=114) \\
n(\%)\end{array}$} & \multicolumn{3}{|c|}{ No PNCS $(n=93)$} \\
\hline & & $n(\%)$ & $\chi^{2}$ & $p$-value \\
\hline Mortality & $5(4.4)$ & $17(18.3)$ & 10.409 & 0.001 \\
\hline LOS & $67(58.8)$ & $4(50.5)$ & 1.404 & 0.148 \\
\hline Necrotising enterocolitis & $4(3.5)$ & $16(17.2)$ & 11.006 & 0.001 \\
\hline Patent ductus arteriosus & $43(37.7)$ & $22(23.7)$ & 4.703 & 0.350 \\
\hline Home oxygen & $3(2.6)$ & $1(1.0)$ & 0.530 & 0.637 \\
\hline NCPAP & $104(91.2)$ & $76(82.6)$ & 3.430 & 0.090 \\
\hline Ventilation & $43(38.1)$ & $39(42.9)$ & 0.484 & 0.566 \\
\hline $\begin{array}{l}\text { Duration of hospitalisation (days), } \\
\text { mean (SD) }\end{array}$ & $62(23.6)$ & $49(16.2)$ & & 0.120 \\
\hline $\begin{array}{l}\text { Duration of ventilation (days), } \\
\text { mean (SD) }\end{array}$ & $17(18.1)$ & $10(10.9)$ & & 0.029 \\
\hline
\end{tabular}

Table 5. Morbidity and ventilatory support on survival for babies with evolving CLD

\begin{tabular}{lllll}
\hline & Survived, $\boldsymbol{n}(\%)$ & Died, $\boldsymbol{n}(\%)$ & $\chi^{2}$ & $\boldsymbol{p}$-value \\
\hline Patent ductus arteriosus & $62 / 199(31.2)$ & $7 / 22(31.8)$ & 0.040 & 1.000 \\
Necrotising enterocolitis & $17 / 212(8.0)$ & $7 / 24(29.2)$ & 10.555 & 0.005 \\
LOS & $114 / 212(53.8)$ & $20 / 24(83.3)$ & 7.676 & 0.008 \\
Surfactant & $183 / 209(87.6)$ & $18 / 23(78.3)$ & 1.548 & 0.206 \\
Ventilation & $83 / 209(39.7)$ & $17 / 24(70.8)$ & 8.510 & 0.004 \\
NCPAP & $178 / 211(84.4)$ & $19 / 24(79.2)$ & 0.429 & 0.557 \\
Duration ventilation (days), mean (SD) & $15.0(16.4)$ & $19.1(13.6)$ & & 0.805 \\
Duration of NCPAP (days), mean (SD) & $5.0(8.0)$ & $9.0(13.2)$ & & 0.0001 \\
$\begin{array}{l}\text { Duration of hospitalisation (days), } \\
\text { mean (SD) }\end{array}$ & $57.3(20.9)$ & $44.4(15.1)$ & & 0.271
\end{tabular}

at CMJAH. Although it was not possible to grade the severity of CLD, most babies were not discharged on oxygen, suggesting that there were few with severe disease. More than $50 \%$ of neonates with CLD at CMJAH received PNCSs, and the use of PNCSs for CLD was associated with improved survival and less NEC. This is contrary to studies that have shown no significant difference in NEC incidence between PNCS and noPNCS use, ${ }^{[15]}$ and, surprisingly, studies have not shown an association between PNCSs and improved survival. ${ }^{[15]}$ The use of PNCSs at CMJAH was $9.1 \%$ of all VLBW infants, which was comparable with that reported in the VON for the same period (2013) at 9.1\%.

Our study findings showed that within the group of babies with evolving CLD, BW did not influence survival, reflecting that at CMJAH most babies who die do so within the first week of life or after 28 days of life when BW has less effect on survival. More ELBW babies are being offered ventilatory support now, and those who survive are at higher risk of developing CLD because of severe prematurity. Our study showed that among these CLD patients, there was a significantly increased rate of LOS and PDAs. These findings are in agreement with a study done by Trembath et al., ${ }^{[20]}$ who outlined the risk factors for CLD.

There is much concern surrounding the use of PNCSs, including sepsis and poor neurological outcome (especially PVL and $\mathrm{CP})$. However, there was no significant difference in LOS in the group that received PNCSs compared with those who did not. Studies have shown an association between use of PNCSs for CLD and PVL. ${ }^{[8,9]}$ The incidence of PVL was very low in the current study and so comparison could not be done. This study only looked at short-term outcome of neonates with CLD and use of PNCSs. Follow-up to evaluate neurodevelopmental outcome in neonates who received PNCSs is necessary. The use of PNCSs for the prevention and treatment of CLD remains controversial. This requires investigation with the aim of improving management (prevention and treatment) of CLD in preterm babies.

\section{Study limitations}

This was a retrospective analysis from an existing database. Information not routinely collected in the database was not readily available. $\mathrm{FiO}_{2}$ was not recorded, so severity of CLD could not be graded. GA was determined by the attending clinicians and may not be accurate, so the definition of 36 weeks PMA may be inaccurate. LOS was classified as culture-proven bacterial or fungal blood stream sepsis only; endotracheal or induced sputa were not available in the database; therefore, the incidence of sepsis may have been underestimated. The definition of CLD requires that the need for oxygen therapy be for a cumulative $\geq 28$ days and not an acute event. Owing to the retrospective nature of the study, it was not possible to distinguish between babies who had been on $\mathrm{O}_{2}$ for a cumulative 28 days and those who had been acutely placed on $\mathrm{O}_{2}$. The type of steroid administered was not recorded.

\section{Conclusions}

CLD remains a common morbidity in VLBW babies despite advances in neonatal care. More ELBW/VLBW babies are surviving. The incidence of CLD is not decreasing. The use of PNCSs has shown short-term benefits in terms of its association with improved survival and reduced NEC. Further studies need to be conducted to determine the safest type of steroid, safe doses and duration of treatment to get the most out of PNCS use for CLD.

\section{References}

1. Northway WH Jr, Rosan RC, Porter DY Pulmonary disease following respiratory therapy of hyaline-membrane disease: Bronchopulmonary dysplasia. N Engl J Med 1967;276(7):357-368. DOI:10.1056/NEJM196702162760701

2. Walsh MC, Yao Q, Gettner P, et al. Impact of physiologic definition on bronchopulmonary dysplasia rates. Pediatrics 2004;114(5):1305-1311.

3. Jobe AH, BancalariE. Bronchopulmonary dysplasia. Am J Respir Crit Care Med 2001;163(7):1723-1729. DOI:10.1164/ajrccm.163.7.2011060

4. Klinger G, Sokolover N, Boyko V, et al. Perinatal risk factors for bronchopulmonary dysplasia in a national cohort of very-low-birth weight infants. Am J Obstet Gynecol 2013;208(2):115.e1-9. DOI:10.1016/j.ajog.2012.11.026

5. Baveja R, Christou H. Pharmacological strategies in the prevention and management of bronchopulmonary dysplasia. Semin Perinatol 2006;30(4):209-218. DOI:10.1053/j.semperi.2006. 05.008

6. Halliday HL, Ehrenkranz RA, Doyle LW. Early postnatal ( $<96$ hours) corticosteroids for preventing chronic lung disease in preterm infants. Cochrane Database Syst Rev 2003;(1):CD001146.

7. HallidayHL, EhrenkranzRA, Doyle LW.Moderately early postnatal (7 - 14 days) corticosteroids for preventing chronic lung disease in preterm infants. Cochrane Database Syst Rev 2003;(1):CD001144. DOI:10.1002/14651858.CD001144

8. Halliday HL, Ehrenkranz RA, Doyle LW. Delayed ( $>3$ weeks) postnatal corticosteroids for preventing chronic lung disease in preterm infants. Cochrane Database Syst Rev 2003;(1):CD001145. DOI:10.1002/14651858.CD001145

9. Terberg AJ, Pena I, Finello K, Angular T, Hodgman JE. Prediction of neurodevelopmental outcome in infants with and without bronchopulmonary dysplasia. Am J Med Sci 1991;301(6):369-374 DOI:10.1097/00000441-199106000-00002 
10. Skidmore MD, Rivers A, Hack M. Increased risk of cerebral palsy among very low birth weight infants with chronic lung disease. Dev Med Child Neurol 1990;32(4):325-331. DOI:10.1111/j.1469-8749.1990.tb16944.x

11. Doyle LW, Halliday HL, Ehrenkranz RA, Davis PG, Sinclair JC. Impact of postnatal systemic corticosteroids on mortality and cerebral palsy in preterm infants: Effect modification by risk for chronic lung disease. Pediatrics 2005;115(3);655-661.

12. Vermont Oxford Network Steroid Study Group. Early postnatal dexamethasone therapy for the prevention of chronic lung disease. Paediatrics 2001;108(3):741-748.

13. Watterberg KL; American Academy of Pediatrics. Committee on Fetus and Newborn. Policy statement - postnatal corticosteroids to prevent or treat bronchopulmonary dysplasia. Pediatrics 2010;126(4):800-808. DOI:10.1542/ peds.2010-1534

14. Yoder BA, Harrison M, Clark RH. Time related changes in steroid use and bronchopulmonary dysplasia in preterm infants. Pediatrics 2009;124(2):673679. DOI:10.1542/peds.2008-2793
15. Vermont Oxford Network, 2014. http://public.vtoxford.org/ (accessed 28 September 2015).

16. Bhandari A, Schramm CM, Kimble C, et al. Effect of a short course of prednisolone in infants with oxygen-dependent bronchopulmonary dysplasia. Pediatrics 2008;121;e344-349. DOI:10.1542/peds.2006-3668

17. Harris PA, Taylor R, Thielke R, et al. Research electronic data capture (REDCap)-A metadata-driven methodology and workflow process for providing translational research informatics support. J Biomed Inform 2009;42(2):377-381. DOI:10.1016/j.jbi.2008.08.010

18. Fenton RT, Kim HJ. A systemic review and meta-analysis to revise the Fenton growth chart for preterm infants. BMC Paediatr 2013;13:59. DOI:10.1186/14712431-13-59

19. Bell MJ, Ternberg JL, Feigin RD, et al. Neonatal necrotizing enterocolitis: Therapeutic decisions based upon clinical staging. Ann Surg 1978;187(1):1-7.

20. Trembath A, Laughon MM. Predictors of bronchopulmonary dysplasia. Clin Perinatol 2012;39(3):585-601. DOI:10.1016/j.clp.2012.06.014 